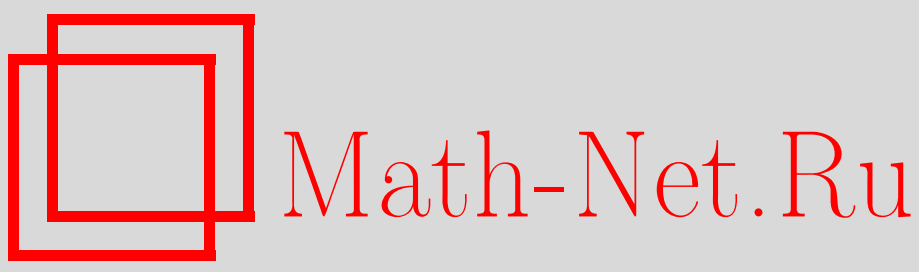

М. Л. Гервер, Е. А. Кудрявцева, Экстремальные свойства дискретных мер, универсальная последовательность и принцип двойственности, Матем. сб., 1998, том 189, номер 11, 3-26

DOI: https://doi.org/10.4213/sm366

Использование Общероссийского математического портала Math-Net.Ru подразумевает, что вы прочитали и согласны с пользовательским соглашением http: //www.mathnet.ru/rus/agreement

Параметры загрузки:

IP : 54.174 .149 .18

26 апреля 2023 г., 18:18:37 


\title{
Экстремальные свойства дискретных мер, универсальная последовательность и принцип двойственности
}

\begin{abstract}
Дается новое, опирающееся на принцип двойственности доказательство теорем об универсальной последовательности и экстремальных свойствах дискретных мер. Эти теоремы ранее были получены авторами другим способом при решении некоторых задач на максимум, возникших в математической геофизике. Переход к двойственньм задачам на минимум раскрьвает геометрический смысл теорем и позволяет получить их обобщения.

Библиография: 10 названий.
\end{abstract}

\section{$\S 1$. Основные результаты}

В статье доказаны и обобщены теоремы, анонсированные в [1], [2].

1.1. Задачи о верхней грани. Универсальная последовательность. На полуоси $s \geqslant 0$ рассмотрим неотрицательные меры Стилтьеса $d a=d a(s)$ с интегралом равньм 1. Каждой такой мере сопоставим функцию

$$
g(d a, t)=\int_{0}^{\infty} \frac{d a(s)}{s+t}, \quad t \geqslant 0 .
$$

Фиксируем неотрицательную меру Стилтьеса $d \theta(t), t \geqslant 0$, и положим

$$
h(d a, x)=\int_{x}^{\infty} g(d a, t) d \theta(t), \quad x \in[0,1] .
$$

Фиксируем положительную функцию (мажоранту) $M=M(t), t \in[0,1]$. Меру $d a$ отнесем к множеству $\mathscr{A}=\mathscr{A}_{M}$, если $g(d a, t) \leqslant M(t)$ при всех $t \in[0,1]$.

На $\mathscr{A}$ рассмотрим 1-параметрическое (зависящее от параметра $x \in[0,1])$ семейство экстремальных задач:

$$
\text { найти } \sup h(d a, x) \text { по всем da } \in \mathscr{A} \text {. }
$$

Физическая трактовка задач (A) дана в [2], [3]. При некоторых ограничениях на $d \theta(t)$ и $M(t)$ в [4] доказано, что при всех $x \in(0,1]$ искомые экстремумы в задачах (A) достигаются на дискретных мерах.

Работа вьполнена при поддержке Российского фонда фундаментальных исследований (грант № 96-01-01852). 
Сформулируем подробнее этот результат. Перепишем формулу для $h(d a, x)$ :

$h(d a, x)=\int_{0}^{\infty} I(s, x) d a(s), \quad I(s, x)=\int_{x}^{\infty} \frac{d \theta(t)}{s+t}, \quad s \geqslant 0, \quad x \in[0,1]$.

В физических приложениях важна мера $d \theta(t)=\frac{2}{\pi} d \sqrt{t}$. В этом случае

$$
I(s, x)=\frac{2}{\pi} \int_{x}^{\infty} \frac{d \sqrt{t}}{s+t},
$$

т.е. $I(s, x)=\frac{2 \operatorname{arctg} \sqrt{s / x}}{\pi \sqrt{s}}, s>0, x>0 ; \quad I(0, x)=\frac{2}{\pi \sqrt{x}} ; \quad I(s, 0)=\frac{1}{\sqrt{s}}$.

Случаю (1.2) посвящены $\S \S 1-4$, общий случай рассмотрен в $\S 5$.

В приложениях мажоранту $M(t)$ нельзя считать непрерьвной. Физически оправдано (см. [2], [4]) предположение: $M(t)$ полунепрерывна снизу в каждой точке $t \in[0,1]$. Всюду далее оно считается выполненным. При этом предположении и дополнительном, тоже физически оправданном, условии

$$
\lim _{t \rightarrow 0} t M(t)=0
$$

в [4] для меры $d \theta(t)=\frac{2}{\pi} d \sqrt{t}$ доказаны следующие теоремы.

ТЕоремА 1 (сушествования и единственности). Для каждого $x \in(0,1] c y$ ществуют и однозначно определяются такое $n=n(x)$ и такой набор чисел

$$
\begin{gathered}
\left\{a_{1}, \ldots, a_{n}, s_{1}, \ldots, s_{n}\right\}, \\
a_{j}=a_{j}(x)>0, \quad 1 \leqslant j \leqslant n, \quad \sum_{j=1}^{n} a_{j}=1, \quad s_{1}=s_{1}(x)>\cdots>s_{n}=s_{n}(x)>0,
\end{gathered}
$$

что строгий максимум $h(d a, x)$ на множестве $\mathscr{A}$ достигается на мере

$$
d a=d a(s)=\sum_{j=1}^{n} a_{j} \delta\left(s-s_{j}\right) d s .
$$

ТЕорема 2 (об универсальной последовательности). Существует такая универсальная (не зависящая от выбора мажсоранты $M(t)$ ) невозрастающая $и$ сходящаяся к нулю последовательность $X_{k} \in[0,1], k \geqslant 1$, что

$$
n(x) \leqslant k \quad \text { при } \quad x \in\left[X_{k}, 1\right] .
$$

В случае (1.2) определение $X_{k}$, данное в [4], связано с полиномами Лежсандра

$$
L_{0}(x)=1, \quad L_{1}(x)=x, \quad L_{n}(x)=\left(2-\frac{1}{n}\right) x L_{n-1}(x)-\left(1-\frac{1}{n}\right) L_{n-2}(x),
$$

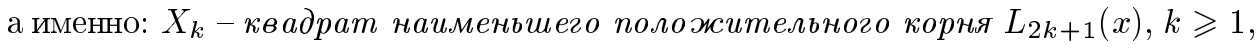

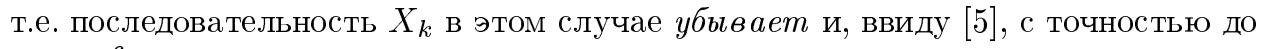
$5 \cdot 10^{-6}$

$$
\begin{aligned}
& X_{1}=0.60000, \quad X_{2}=0.28995, \quad X_{3}=0.16471, \quad X_{4}=0.10514, \\
& X_{5}=0.07265, \quad X_{6}=0.05311, \quad X_{7}=0.04048, \quad X_{8}=0.03186, \\
& X_{9}=0.02572, \quad X_{10}=0.02119, \quad X_{11}=0.01776, \quad X_{12}=0.01510, \ldots .
\end{aligned}
$$

В общем случае ограничения на $d \theta(t)$ и определение $X_{k}$ cм. в $\S 5$. 
1.2. Двойственные экстремальные задачи. В статье будет дано новое, опираюшееся на принцип двойственности доказательство теорем 1,2 .

Рассмотрим пары $\left(b_{0}, d b=d b(t)\right)$, где $b_{0}$ - неотрицательные числа, a $d b(t)-$ неотрицательные меры Стилтьеса на отрезке [0,1]. Множество всех таких пар обозначим через $\mathscr{P}$.

Каждой паре из $\mathscr{P}$ сопоставим функцию $r\left(b_{0}, d b, s\right), s \geqslant 0$, и число $H\left(b_{0}, d b\right)$ :

$$
r\left(b_{0}, d b, s\right)=b_{0}+\int_{0}^{1} \frac{d b(t)}{s+t}, \quad H\left(b_{0}, d b\right)=b_{0}+\int_{0}^{1} M(t) d b(t) .
$$

На полуоси $s \geqslant 0$ фиксируем 1-параметрическое (зависяшее от параметра $x$ ) $c e$ мейство минорант $I(s, x)$ (см. (1.2)). Зафиксируем $x \in[0,1]$ в (1.1) и (1.2), положим $h(d a)=h(d a, x), I(s)=I(s, x), s \geqslant 0$, и отнесем к множеству $\mathscr{B}=\mathscr{B}(x)$ пары $\left(b_{0}, d b\right) \in \mathscr{P}$, для которых $r\left(b_{0}, d b, s\right) \geqslant I(s)$ при всех $s \geqslant 0$.

Задача, двойственная к (А), формулируется так:

$$
\text { найтu } \inf H\left(b_{0}, d b\right) \text { по всем }\left(b_{0}, d b\right) \in \mathscr{B}(x) \text {. }
$$

TЕОРемА 3 (принцип двойственности). В задачах (А) $и$ (В) верхняя и нижсняя грани достигаются и равнь друг другу при любом $x \in(0,1]$ :

$$
\max _{\mathscr{A}} h(d a, x)=\min _{\mathscr{B}(x)} H\left(b_{0}, d b\right), \quad x \in(0,1] .
$$

Доказательство теоремы 3 (приведенное в $\S 3$ ) базируется на следующей - совершенно прозрачной - геометрической конструкции.

Пусть $V$ - вьпуклое множество в $\mathbb{R}^{n}$, расположенное над некоторой гиперплоскостью. Пусть ориентированная (направленная вверx) прямая $L$ пересекает $V$. Обозначим через $H$ нижнюю грань точек множества $V$ на $L$ и через $h$ - верхнюю грань точек прямой $L$, принадлежащих всевозможным гиперплоскостям, трансверсальным к $L$ и лежашим под $V$. Пусть гиперплоскость, опорная $\kappa V$ в точке $H$, трансверсальна $\kappa L$ (если таких гиперплоскостей более одной, пусть указанньм свойством обладает хоть одна из них). Тогда, очевидно,

$$
h=H .
$$

1.3. Особые точки. План доказательства теорем $1,2$. При проверке неравенств (1.4) используется

ОПреДЕлЕНИЕ. Особъми точками меры $d a \in \mathscr{A}$ назовем точки $t \in[0,1]$, в которых $g(d a, t)=M(t)$, а особыми точками пары $\left(b_{0}, d b\right) \in \mathscr{B}-$ точки $s \geqslant 0$, в которых $r\left(b_{0}, d b, s\right)=I(s)$.

В $§ 2$ будут доказаны следующие утверждения.

КРИТЕРИЙ СОВПАДЕНИЯ $h(d a)$ и $H\left(b_{0}, d b\right)$. Для любой меры $d a \in \mathscr{A}$ и любой пары $\left(b_{0}, d b\right) \in \mathscr{B}$ выполняется неравенство $h(d a) \leqslant H\left(b_{0}, d b\right)$. Для совпадения $h(d a)$ и $H\left(b_{0}, d b\right)$ необходимо и достаточно, чтобъ мера dа бъла сосредоточена в особъх точках $\left(b_{0}, d b\right)$, а мера $d b$ - в особъх точках $d a$. 
ОСНОВНОЕ УТВЕРЖДЕНИЕ ОБ ОСОБЫХ ТОЧКАХ. Пусть $x \geqslant X_{k} u з$ (1.5) $u$

$$
s_{j}, \quad 1 \leqslant j \leqslant m, \quad s_{1}>\cdots>s_{m} \geqslant 0,
$$

являются особьми точками пары $\left(b_{0}, d b\right) \in \mathscr{B}=\mathscr{B}(x)$. Тогда $m \leqslant k$.

Из последнего неравенства, принципа двойственности и критерия совпадения $h(d a)$ и $H\left(b_{0}, d b\right)$ следует, что мера $d a^{\circ}$, на которой достигается $\max _{\mathscr{A}} h(d a, x)$, дискретна при $x \in(0,1]$ и сосредоточена максимум в $k$ точках при $x \geqslant X_{k}$ :

Теорема 4 (о дискретности $d a^{\circ}$ ). Пусть $x \geqslant X_{k} u з$ (1.5) $u \max _{\mathscr{A}} h(d a, x)$ достигается на мере $d a^{\circ}$. Тогда $d a^{\circ}$ сосредоточена в конечном числе точек $s_{1}^{\circ}>\cdots>s_{n}^{\circ} \geqslant 0$ un $=n(x) \leqslant k$.

В $\S 4$ будет доказана

Теорема 5 (о строгом максимуме). Для любой меры $d a \in \mathscr{A}$, не равной $d a^{\circ}$, выполняется строгое неравенство $h(d a)<h\left(d a^{\circ}\right)$.

Нетрудно проверить, что при условии (1.3) $s_{n}^{\circ}$ в теореме 4 положительно: $s_{n}^{\circ}>0$. Тем самым, теоремы 1 и 2 являются следствиями теорем 3-5.

\section{§ 2. Основное утверждение об особых точках}

2.1. Реализацию плана, намеченного в п. 1.3, начнем с доказательства леммы, эквивалентной критерию совпадения $h(d a)$ и $H\left(b_{0}, d b\right)$. В ней

$$
h(d a)=h(d a, x), \quad I(s)=I(s, x), \quad \mathscr{B}=\mathscr{B}(x),
$$

где $x \in[0,1]$ - произвольно фиксированное значение параметра $x$.

Лемма 1. Для любой мерьц $d a \in \mathscr{A}$ и любой пары $\left(b_{0}, d b\right) \in \mathscr{B}$

$$
h(d a) \leqslant H\left(b_{0}, d b\right)
$$

причем равенство достигается в том и только том случае, когда

$$
\int_{0}^{\infty}\left[r\left(b_{0}, d b, s\right)-I(s)\right] d a(s)=0 \quad u \quad \int_{0}^{1}[M(t)-g(d a, t)] d b(t)=0 .
$$

ДокАЗАТЕЛьСтво. Согласно (1.1)

$$
h(d a)=\int_{0}^{\infty} I(s) d a(s) .
$$

Поэтому с учетом (1.6) лемма 1 вытекает из следуюшей цепочки неравенств:

$$
\begin{aligned}
h(d a) & =\int_{0}^{\infty} I(s) d a(s) \leqslant \int_{0}^{\infty} r\left(b_{0}, d b, s\right) d a(s) \\
& =\int_{0}^{\infty}\left[b_{0}+\int_{0}^{1} \frac{d b(t)}{s+t}\right] d a(s)=b_{0}+\int_{0}^{1} \int_{0}^{\infty} \frac{d a(s) d b(t)}{s+t} \\
& =b_{0}+\int_{0}^{1} g(d a, t) d b(t) \leqslant b_{0}+\int_{0}^{1} M(t) d b(t)=H\left(b_{0}, d b\right) .
\end{aligned}
$$


ЗАмечАнИЕ. Из (2.1) следует (ср. с (1.7)), что

$$
\sup _{\mathscr{A}} h(d a) \leqslant \inf _{\mathscr{B}} H\left(b_{0}, d b\right) .
$$

\section{2. Теперь докажем}

ОСНовное УТвЕРЖ ДЕНИЕ ОБ ОСОБЫХ ТОчкаХ. При $x \geqslant X_{k}$ из (1.5) любая пара $\left(b_{0}, d b\right) \in \mathscr{B}=\mathscr{B}(x)$ имеет максимум $k$ особых точек.

ДоказАтельство. Допустим, что это утверждениеневерно: что, хотя $x \geqslant X_{k}$, существует пара $\left(b_{0}, d b\right) \in \mathscr{B}(x)$, имеющая по крайней мере $k+1$ особых точек:

$$
r\left(b_{0}, d b, s_{j}\right)=I\left(s_{j}\right), \quad 1 \leqslant j \leqslant k+1, \quad s_{1}>\cdots>s_{k+1} \geqslant 0
$$

и приведем это допущение к противоречию. Предложим сначала набросок доказательства, отметив значками $\langle n\rangle, 1 \leqslant n \leqslant 5$, утверждения, требующие более подробных пояснений, а потом дадим такие пояснения.

Напомним определение $I(s)$ : мы зафиксировали $x \in[0,1]$ и положили $I(s)=$ $I(s, x), s \geqslant 0$, таким образом:

$$
I(s)=\int_{x}^{\infty} \frac{d \theta(t)}{s+t}, \quad d \theta(t)=\frac{2}{\pi} d \sqrt{t}, \quad 0<t<\infty
$$

Согласно [6] при любом $x>0$ сушествуют и единственны такие положительные числа

$$
p_{0}, p_{1}, \ldots, p_{k}, \quad t_{1}, \ldots, t_{k}, \quad t_{1}>\cdots>t_{k}>x>0,
$$

что функция

$$
p(s)=p_{0}+\sum_{i=1}^{k} \frac{p_{i}}{s+t_{i}}
$$

вместе с производной $p^{\prime}(s)$ совпадает с $I(s)$ (соответственно $\left.I^{\prime}(s)\right)$ в точках $s_{j}$, $1 \leqslant j \leqslant k$, а также при $s=0\langle 1\rangle$ :

$$
p\left(s_{j}\right)=I\left(s_{j}\right), \quad p^{\prime}\left(s_{j}\right)=I^{\prime}\left(s_{j}\right), \quad 1 \leqslant j \leqslant k, \quad p(0)=I(0) .
$$

Сравнивая $p(s)$ с $r\left(b_{0}, d b, s\right)$, нетрудно доказать, что $t_{1}<1\langle 2\rangle$.

Однако из условия $x \geqslant X_{k}$ согласно [4] следует, что $t_{1}>1$. Это противоречие доказывает основное утверждение об особых точках.

Доказательство неравенства $t_{1}>1$ основывается на следуюшем характеристическом свойстве последовательности $X_{k}$. При любом $x>0$ сушествует и единственна пара

$$
\left(b_{00}, d b_{0}\right) \in \mathscr{B}(x), \quad d b_{0}=\sum_{i=1}^{k} b_{0 i} \delta\left(t-t_{0 i}\right) d t, \quad t_{01}>\cdots>t_{0 k}>0,
$$


для которой при $s=0$ функция

$$
r_{0}(s)=r\left(b_{00}, d b_{0}, s\right)=b_{00}+\sum_{i=1}^{k} \frac{b_{0 i}}{s+t_{0 i}}
$$

совпадает с $I(s)=I(s, x)$ вместе с $2 k$ производньми ${ }^{\langle 3\rangle}$ :

$$
r_{0}^{(j)}(0)=I^{(j)}(0), \quad 0 \leqslant j \leqslant 2 k ;
$$

при этом соотношения $t_{01}<1, t_{01}=1$ и $t_{01}>1$ эквивалентны (соответственно) соотношениям $x<X_{k}, x=X_{k}$ и $x>X_{k}\langle 4\rangle$.

Таким образом, достаточно проверить, что $t_{1}$ в $(2.5),(2.6)$ и $t_{01}$ в $(2.8)$ связаны неравенством $t_{1}>t_{01}\langle 5\rangle$. Тогда $t_{1}>t_{01} \geqslant 1$ при $x \geqslant X_{k}$.

\section{Пояснения к $\S 2$}

Пояснение 1. Сушествование и единственность функции $p(s)$ вида (2.6), определяемой параметрами (2.5) и связанной с $I(s)$ равенствами $(2.7)$, допускает красивую геометрическую интерпретацию, опирающуюся на теорему $\Phi$. Рисса о замкнутой выпуклой оболочке кривой в $\mathbb{R}^{n}$ и центрах масс [6; гл. 1 , теорема 3.5$]$.

Перепишем (2.4) в виде

$$
I(s)=\int_{x}^{\infty} \frac{t d \rho(t)}{s+t}, \quad d \rho(t)=\frac{d \theta(t)}{t}=\frac{1}{\pi} d\left(\frac{-1}{\sqrt{t}}\right), \quad 0<x \leqslant t<\infty,
$$

и положим $J(s)=\pi \sqrt{x} I(s)$; множитель $\pi \sqrt{x}$ выбран так, чтобы в формуле

$$
J(s)=\int_{x}^{\infty} \frac{t d \mu(t)}{s+t}, \quad d \mu(t)=\pi \sqrt{x} d \rho(t)=d\left(-\frac{\sqrt{x}}{\sqrt{t}}\right), \quad 0<x \leqslant t<\infty,
$$

для меры $d \mu(t)$ вьполнялось равенство:

$$
\int_{x}^{\infty} d \mu(t)=1
$$

Пусть

$$
\xi(t)=\left\{\xi_{0}=1, \frac{t}{s_{1}+t}, \frac{-t}{\left(s_{1}+t\right)^{2}}, \ldots, \frac{t}{s_{k}+t}, \frac{-t}{\left(s_{k}+t\right)^{2}}\right\},
$$

так что точка $C=\left\{J(0), J\left(s_{1}\right), J^{\prime}\left(s_{1}\right), \ldots, J\left(s_{k}\right), J^{\prime}\left(s_{k}\right)\right\} \in \mathbb{R}^{2 k+1}$ представима в виде

$$
C=\left\{J(0), J\left(s_{1}\right), J^{\prime}\left(s_{1}\right), \ldots, J\left(s_{k}\right), J^{\prime}\left(s_{k}\right)\right\}=\int_{x}^{\infty} \xi(t) d \mu(t) .
$$

Вектор-функция $\xi(t), t \in[x, \infty]$, задает дугу $\mathscr{D}$ в $\mathbb{R}^{2 k+1}$, лежашую в гиперплоскости $\xi_{0}=1$ и содержашую точку $B=\xi(\infty)=\left\{\xi_{0}=1,1,0, \ldots, 1,0\right\}$.

Замкнутую вьпуклую оболочку дуги $\mathscr{D}$ обозначим через $\mathscr{R}(\mathscr{D})$. По теореме $\Phi$. Рисса $\mathscr{R}(\mathscr{D})$ совпадает с множеством точек $P$, допускающих представление

$$
P=\int_{x}^{\infty} \xi(t) d m(t), \quad \int_{x}^{\infty} d m(t)=1,
$$


т.е. совпадает с множеством центров масс для всевозможных распределений $m(t)$ неотрицательных масс на дуге $\mathscr{D}$.

В частности, можно взять $m(t)=\mu(t)=1-\sqrt{x / t}$. Тем самьм,

$$
C=\left\{J(0), J\left(s_{1}\right), J^{\prime}\left(s_{1}\right), \ldots, J\left(s_{k}\right), J^{\prime}\left(s_{k}\right)\right\} \in \mathscr{R}(\mathscr{D})
$$

Спрашивается: можно ли так подобрать $t_{1}, \ldots, t_{k}, t_{1}>\cdots>t_{k}>x>0, u$ поместить в точку $\xi(\infty)$ и в точки $\xi\left(t_{i}\right)$ такие положстельные массь $m_{\infty}$ u $m_{i}, 1 \leqslant i \leqslant k, m_{\infty}+m_{1}+\cdots+m_{k}=1$, чтобъ иентр этой системы масс coвnал $c C$ ? Утвердительньй ответ на этот вопрос означает, что

$$
\left\{J(0), J\left(s_{1}\right), J^{\prime}\left(s_{1}\right), \ldots, J\left(s_{k}\right), J^{\prime}\left(s_{k}\right)\right\}=m_{\infty} \xi(\infty)+m_{1} \xi\left(t_{1}\right)+\cdots+m_{k} \xi\left(t_{k}\right)
$$

последнее соотношение, как нетрудно проверить, эквивалентно равенствам

$$
\begin{gathered}
J(0)=m_{\infty}+m_{1}+\cdots+m_{k}=1, \\
J\left(s_{j}\right)=m_{\infty}+\frac{m_{1} t_{1}}{s_{j}+t_{1}}+\cdots+\frac{m_{k} t_{k}}{s_{j}+t_{k}}, \quad 1 \leqslant j \leqslant k \\
J^{\prime}\left(s_{j}\right)=\frac{-m_{1} t_{1}}{\left(s_{j}+t_{1}\right)^{2}}+\cdots+\frac{-m_{k} t_{k}}{\left(s_{j}+t_{k}\right)^{2}}, \quad 1 \leqslant j \leqslant k,
\end{gathered}
$$

и равенства (2.7) следуют из них, если положить

$$
p_{0}=\frac{m_{\infty}}{\pi \sqrt{x}}, \quad p_{i}=\frac{m_{i} t_{i}}{\pi \sqrt{x}}, \quad 1 \leqslant i \leqslant k
$$

Расположить на дуге $\mathscr{D}$ конечное число масс с иентром в заданной точке $C \in \mathscr{R}(\mathscr{D})$ не представляет труда. Тонкость состоит в том, что масс должно быть всего $k+1$, причем одна из них должна располагаться в точке $B=\xi(\infty)$.

Обойтись столь небольшим числом масс можно благодаря тому, что компоненты вектор-функции $\xi(t)$, задающей дугу $\mathscr{D}$,

$\xi_{0}=1, \xi_{1}(t)=\frac{t}{s_{1}+t}, \xi_{2}(t)=\frac{-t}{\left(s_{1}+t\right)^{2}}, \ldots, \xi_{2 k-1}(t)=\frac{t}{s_{k}+t}, \xi_{2 k}(t)=\frac{-t}{\left(s_{k}+t\right)^{2}}$

образуют чебышёвскую систему (или Т-систему) порядка $2 k$. По определению (см. [6]-[8]) это означает, что любая нетривиальная линейная комбинация (или, как принято говорить, любой нетривиальньй многочлен) $\sum_{j=0}^{2 k} c_{j} \xi_{j}(t)$ имеет на полуоси $t>0$ самое большее $2 k$ корней.

Итак, докажем существование и единственность системь из $k+1$ масс на дуге $\mathscr{D}$ с одной из масс в точке $B=\xi(\infty)$ и с иентром масс $C$ (ср. с аналогичными рассуждениями в $[9 ; \S 6$, п. $6.1, \S 8$, комментарий 8$])$.

Проведем луч $B C$. Пусть $E$ - точка, в которой он выходит из $\mathscr{R}(\mathscr{D})$, и $\Gamma$ - опорная гиперплоскость к $\mathscr{R}(\mathscr{D})$ в точке $E$.

До сих пор построение проводилось в аффинном подпространстве $\xi_{0}=1$ в $\mathbb{R}^{2 k+1}$, так что $\operatorname{dim} \Gamma=2 k-1$. Теперь возьмем линейное подпространство $\Lambda$ 
в $\mathbb{R}^{2 k+1}(\operatorname{dim} \Lambda=2 k)$, содержащее $\Gamma: \lambda_{0} \xi_{0}+\lambda_{1} \xi_{1}+\cdots+\lambda_{2 k} \xi_{2 k}=0$; ориентацию выберем так, чтобы для точек дуги $\mathscr{D}$

$$
\xi(t)=\left\{\xi_{j}(t)\right\}_{j=0}^{2 k}, \quad t \in[x, \infty],
$$

лежащих по одну сторону от $\Lambda$, многочлен $\lambda(t)=\sum_{j=0}^{2 k} \lambda_{j} \xi_{j}(t)$ был неотрицателен (на $\mathscr{D} \cap \Gamma$ он равен нулю).

Представим $E$ как центр масс на дуге $\mathscr{D}$. Так как $E \in \mathscr{R}(\mathscr{D}) \cap \Gamma$, то все эти массы расположены в точках $\mathscr{D} \cap \Gamma$.

Таких точек - конечное число $q$ (не больше чем корней $\lambda(t)$ ), т.е.

$$
E=\sum_{i=1}^{q} e_{i} \xi\left(t_{i}\right), \quad \sum_{i=1}^{q} e_{i}=1, e_{i}>0,1 \leqslant i \leqslant q, t_{1}>\cdots>t_{q} \geqslant x>0
$$

Докажем, что $q=k$. Неравенство $q>k$ невозможно, так как в этом случае число корней многочлена $\lambda(t)-\varepsilon$ (при малом $\varepsilon>0$ ) больше чем $2 k$.

Чтобы проверить, что неравенство $q<k$ тоже невозможно, воспользуемся теоремой, которая распространяет на случай обобщенных чебышёвских систем (или ЕТ-систем [7]) классическое неравенство $K \leqslant \Pi$, связьвающее сумму $K$ кратностей положительных корней многочлена с числом П перемен знака в последовательности его коэффициентов.

Будем называть ее теоремой Гантмахера-Крейна (хотя, если быть более точными, это - ее модификация (см. [6; гл. $2, \S 2$, Д. 2.3], [7; гл. 1, теорема 4.4] или [8]).

Допустим, что $q<k$, и приведем это допущение к противоречию.

Представим $C$ в виде $C=m B+(1-m) E, m \in[0,1]$ (из дальнейшего ясно, что $m \in(0,1))$. Полагая

$$
m_{\infty}=m, \quad m_{i}=(1-m) e_{i}, \quad 1 \leqslant i \leqslant q,
$$

ввиду (2.10) получаем: $C=m_{\infty} \xi(\infty)+m_{1} \xi\left(t_{1}\right)+\cdots+m_{q} \xi\left(t_{q}\right)$, т.е. вместо $(2.6)$

$$
p(s)=p_{0}+\sum_{i=1}^{q} \frac{p_{i}}{s+t_{i}}, \quad q<k, \quad t_{1}>\cdots>t_{q} \geqslant x>0
$$

Пусть $t_{q}>x$ (случай $t_{q}=x$, как видно из последующего доказательства, невозможен даже при $q=k$ ). Функцию $I(s)$ (см. (2.4)) представим в виде суммы $q+1$ слагаемых: $I(s)=I_{0}(s)+I_{1}(s)+\cdots+I_{q}(s)$, где

$$
I_{q}(s)=\int_{x}^{t_{q}} \frac{d \theta(t)}{s+t}, \ldots, I_{1}(s)=\int_{t_{2}}^{t_{1}} \frac{d \theta(t)}{s+t}, I_{0}(s)=\int_{t_{1}}^{\infty} \frac{d \theta(t)}{s+t}, d \theta(t)=\frac{2}{\pi} d \sqrt{t} .
$$

При $q \leqslant k-1$ число П перемен знака в разложении разности

$$
p(s)-I(s)=p_{0}-I_{0}(s)+\frac{p_{1}}{s+t_{1}}-I_{1}(s)+\cdots+\frac{p_{q}}{s+t_{q}}-I_{q}(s)
$$


может оказаться равным самое большее $2 k-1$. Однако по теореме Гантмахера-Крейна сумма $K$ кратностей корней $p(s)-I(s)$ не больше П. При этом вследствие (2.7) $K \geqslant 2 k+1$.

Полученное противоречие доказывает, что неравенство $q<k$ невозможно и, следовательно, $q=k$ (причем $t_{1}>\cdots>t_{k}>x>0$ ).

Единственность найденной системы из $k+1$ масс очевидна: если бы две функции $p_{1}(s)$ и $p_{2}(s)$ вида (2.6) удовлетворяли $(2.7)$, то их разность

$$
p_{1}(s)-p_{2}(s)=p_{01}-p_{02}+\sum_{i=1}^{k} \frac{p_{i 1}}{s+t_{i 1}}-\sum_{i=1}^{k} \frac{p_{i 2}}{s+t_{i 2}}
$$

имела бы слишком много корней-вследствие (2.7) сумма их кратностей не меньше $2 k+1$, в то время как максимально возможное число корней $(2.11)$ с учетом кратности - лишь $2 k$.

Пояснение 2. Для проверки неравенства $t_{1}<1$ снова воспользуемся теоремой Гантмахера-Крейна. Если в $(2.5) t_{1}<1$, то функцию

$$
r(s)=r\left(b_{0}, d b, s\right)=b_{0}+\int_{0}^{1} \frac{d b(t)}{s+t}, \quad s \geqslant 0
$$

можно представить в виде суммы $2 k+2$ слагаемых:

$$
r(s)=\int_{0}^{t_{k}-0} \frac{d b(t)}{s+t}+\frac{b_{k}}{s+t_{k}}+\cdots+\int_{t_{2}+0}^{t_{1}-0} \frac{d b(t)}{s+t}+\frac{b_{1}}{s+t_{1}}+\int_{t_{1}+0}^{1} \frac{d b(t)}{s+t}+b_{0},
$$

где

$$
\frac{b_{i}}{s+t_{i}}=\int_{t_{i}-0}^{t_{i}+0} \frac{d b(t)}{s+t}, \quad 1 \leqslant i \leqslant k
$$

Тем самым число П перемен знака в разложении разности $p(s)-r(s)$

$$
\left(p_{0}-b_{0}\right)-\int_{t_{1}+0}^{1} \frac{d b(t)}{s+t}+\frac{p_{1}-b_{1}}{s+t_{1}}-\int_{t_{2}+0}^{t_{1}-0} \frac{d b(t)}{s+t}+\cdots+\frac{p_{k}-b_{k}}{s+t_{k}}-\int_{0}^{t_{k}-0} \frac{d b(t)}{s+t}
$$

может оказаться равным самое большее $2 k+1$. По теореме Гантмахера-Крейна сумма $K$ кратностей корней $p(s)-r(s)$ не больше числа П. Легко проверить, что $2 k+1 \leqslant K$ (мы обоснуем это неравенство чуть ниже). Таким образом, в случае $t_{1}<1$ неравенство $K \leqslant$ П обращается в равенство

$$
2 k+1=K=\Pi \text {. }
$$

Если в (2.5) $t_{1}=1$, то

$$
r(s)=\int_{0}^{t_{k}-0} \frac{d b(t)}{s+t}+\frac{b_{k}}{s+t_{k}}+\cdots+\int_{t_{2}+0}^{t_{1}-0} \frac{d b(t)}{s+t}+\frac{b_{1}}{s+t_{1}}+b_{0}
$$

и в разложении разности

$$
p(s)-r(s)=\left(p_{0}-b_{0}\right)+\frac{p_{1}-b_{1}}{s+t_{1}}-\int_{t_{2}+0}^{t_{1}-0} \frac{d b(t)}{s+t}+\cdots+\frac{p_{k}-b_{k}}{s+t_{k}}-\int_{0}^{t_{k}-0} \frac{d b(t)}{s+t}
$$


число П перемен знака меньше чем $2 k+1$, т.е. заведомо меньше чем $K$, вопреки теореме Гантмахера-Крейна, согласно которой $K \leqslant \Pi$.

Тот же вывод тем более верен, если $t_{1}>1$.

Таким образом, остается обосновать неравенство $2 k+1 \leqslant K$. Так как

$$
r(s)=r\left(b_{0}, d b, s\right) \geqslant I(s), \quad s \geqslant 0, \quad r\left(s_{j}\right)=I\left(s_{j}\right), \quad 1 \leqslant j \leqslant k+1,
$$

то $r\left(s_{j}\right)=I\left(s_{j}\right), r^{\prime}\left(s_{j}\right)=I^{\prime}\left(s_{j}\right), 1 \leqslant j \leqslant k$. Следовательно, ввиду $(2.7)$

$$
r\left(s_{j}\right)=p\left(s_{j}\right), \quad r^{\prime}\left(s_{j}\right)=p^{\prime}\left(s_{j}\right), \quad 1 \leqslant j \leqslant k .
$$

Рассмотрим два случая: 1) $r(0)=p(0)=I(0)$ и 2) $r(0)>p(0)=I(0)$, а значит, $s_{k+1}>0$ и тем самьм $r\left(s_{k+1}\right)=I\left(s_{k+1}\right)<p\left(s_{k+1}\right)$. В обоих случаях кроме $k$ кратных корней $s_{j}, 1 \leqslant j \leqslant k$, разность $p(s)-r(s)$ имеет еше хотя бы один корень ( $s=0$ в первом случае и $s=s^{0} \in\left(0, s_{k+1}\right)$ - во втором).

Проверка неравенства $t_{1}<1$ закончена.

ЗАмЕчАниЕ. Далее в этой статье мы еще несколько раз будем опираться в своих доказательствах на теорему Гантмахера-Крейна.

Пояснение 3. Возьмем в (1.2) $x=1$, положим

$$
\widehat{I}(s)=I(s, 1)=\frac{2 \operatorname{arctg} \sqrt{s}}{\pi \sqrt{s}}=\frac{2}{\pi} \int_{1}^{\infty} \frac{d \sqrt{t}}{s+t}, \quad s \geqslant 0,
$$

и построим рациональную аппроксимацию $\hat{r}_{0}(s)$ функции $\widehat{I}(s)$ :

$$
\begin{gathered}
\hat{r}_{0}(s)=\hat{b}_{00}+\sum_{i=1}^{k} \frac{\hat{b}_{0 i}}{s+\hat{t}_{0 i}}, \quad \hat{t}_{01}>\cdots>\hat{t}_{0 k}>0, \\
\hat{r}_{0}^{(j)}(0)=\widehat{I}^{(j)}(0), \quad 0 \leqslant j \leqslant 2 k .
\end{gathered}
$$

ЗАмечаниЕ. Сравнивая (1.2) и (2.12), видим, что $I(s)=\widehat{I}(s / x) / \sqrt{x}$. Значит, рациональные аптроксимации $r_{0}(s)$ и $\hat{r}_{0}(s)$ связаны соотношением

$$
r_{0}(s)=\frac{\hat{r}_{0}(s / x)}{\sqrt{x}}=\frac{1}{\sqrt{x}}\left(\hat{b}_{00}+\sum_{i=1}^{k} \frac{\hat{b}_{0 i}}{s / x+\hat{t}_{0 i}}\right)=\frac{\hat{b}_{00}}{\sqrt{x}}+\sum_{i=1}^{k} \frac{\hat{b}_{0 i} \sqrt{x}}{s+\hat{t}_{0 i} x} .
$$

Таким образом, чтобы доказать существование и единственность аппроксимации $r_{0}(s)$, достаточно построить аппроксимацию $\hat{r}_{0}(s)$ и проверить, что она однозначно определяется условиями (2.14).

При построении $\hat{r}_{0}(s)$ перед нами возникнет следующая

ЗАДАчА. Найти $c_{1}, \ldots, c_{k}$ и $\zeta_{1}, \ldots, \zeta_{k}$, удовлетворяющие нелинейной системе уравнений

$$
\sum_{i=1}^{k} c_{i} \zeta_{i}^{p}=\frac{1}{2 p+3}, \quad 0 \leqslant p \leqslant 2 k-1 .
$$

В $[4 ; \S 6$, теорема $8, \S 12$, комментарий 14$]$ доказана 
Tеорема 6. Система (2.16) однозначно разрешима, причем $\zeta_{1}, \ldots, \zeta_{k}-$ это квадраты ненулевых корней полинома Лежсандра $L_{2 k+1}(x)$, a $c_{1}, \ldots, c_{k}$ определяются из системы линейных уравнений

$$
\sum_{i=1}^{k} c_{i} \zeta_{i}^{p}=\frac{1}{2 p+3}, \quad 0 \leqslant p \leqslant k-1
$$

Опираясь на эту теорему, построим $\hat{r}_{0}(s)$. Из (2.12) и (2.13) следует, что

$$
\widehat{I}(0)=\frac{2}{\pi}, \quad \hat{r}_{0}(0)=\hat{b}_{00}+\sum_{i=1}^{k} \frac{\hat{b}_{0 i}}{\hat{t}_{0 i}}
$$

и что при $1 \leqslant j \leqslant 2 k$

$$
\frac{(-1)^{j}}{j !} \widehat{I}^{(j)}(0)=\frac{2}{\pi} \int_{1}^{\infty} \frac{d \sqrt{t}}{t^{j+1}}, \quad \frac{(-1)^{j}}{j !} \hat{r}^{(j)}(0)=\sum_{i=1}^{k} \frac{\hat{b}_{0 i}}{\hat{t}_{0 i}^{j+1}} .
$$

Поэтому условия (2.14) эквивалентны системе уравнений

$$
\hat{b}_{00}+\sum_{i=1}^{k} \frac{\hat{b}_{0 i}}{\hat{t}_{0 i}}=\frac{2}{\pi}, \quad \sum_{i=1}^{k} \frac{\hat{b}_{0 i}}{\hat{t}_{0 i}^{j+1}}=\frac{2}{\pi} \int_{1}^{\infty} \frac{d \sqrt{t}}{t^{j+1}}, \quad 1 \leqslant j \leqslant 2 k,
$$

относительно $\hat{b}_{00}$ и $\hat{b}_{0 i}, \hat{t}_{0 i}, 1 \leqslant i \leqslant k$. Если положить

$$
\zeta_{i}=\frac{1}{\hat{t}_{0 i}}, \quad c_{i}=\frac{\pi}{2} \frac{\hat{b}_{0 i}}{\hat{t}_{0 i}^{2}}, \quad 1 \leqslant i \leqslant k ; \quad u=\frac{1}{t}, \quad \nu(u)=\frac{u^{3 / 2}}{3},
$$

то последние $2 k$ уравнений из (2.17) принимают вид

$$
\sum_{i=1}^{k} c_{i} \zeta_{i}^{p}=\int_{0}^{1} u^{p} d \nu(u), \quad 0 \leqslant p \leqslant 2 k-1 .
$$

Вычислив интегралы, приходим к системе уравнений

$$
\sum_{i=1}^{k} c_{i} \zeta_{i}^{p}=\frac{1}{2 p+3}, \quad 0 \leqslant p \leqslant 2 k-1
$$

т.е. оказываемся в условиях теоремы 6. Добавляя первое уравнение из (2.17), получаем, что аппроксимация $\hat{r}_{0}(s)$ определяется однозначно условиями (2.14).

Пояснение 4. Занумеруем $\zeta_{i}$ так, что $0<\zeta_{1}<\cdots<\zeta_{k}$, тогда (по теореме 6) $\zeta_{1}=X_{k}$ - квадрат наименьшего положительного корня полинома Лежандра $L_{2 k+1}$, т.е.

$$
\hat{t}_{01}=\frac{1}{X_{k}} .
$$

Вследствие (2.15) $t_{0 i}=\hat{t}_{0 i} x, 1 \leqslant i \leqslant k$, откуда с учетом (2.18) $t_{01}=x / X_{k}$. Итак, соотношения $t_{01} \leqslant 1, t_{01}=1$ и $t_{01} \geqslant 1$ вьполняются соответственно при $x \leqslant X_{k}$, $x=X_{k}$ и $x \geqslant X_{k}$. 
Пояснение 5. Разобьем доказательство неравенства $t_{1}>t_{01}$ на три части.

А. Для функции $p(s)$ из (2.6) введем обозначение $p(\mathscr{S}, s)$, где $\mathscr{S}=\left\{s_{1}, \ldots, s_{k}\right\}$ - набор точек на полуоси $s>0$, в которых (в соответствии с (2.7)) $p(s)$ совпадает с $I(s)$ вместе с производной. Аналогично, $p^{*}(s)=p\left(\mathscr{S}^{*}, s\right)$ - это функция вида (2.6), совпадаюшая с $I(s)$ вместе с производной в точках $s_{j}^{*}, 1 \leqslant j \leqslant k$, из набора $\mathscr{S}^{*}=\left\{s_{1}^{*}, \ldots, s_{k}^{*}\right\}, s_{1}^{*}>\cdots>s_{k}^{*}>0$. Кроме того, как и в $(2.7), p^{*}(0)=I(0)$. Пусть точки наборов $\mathscr{S}$ и $\mathscr{S}^{*}$ чередуются:

$$
s_{1} \geqslant s_{1}^{*} \geqslant \cdots \geqslant s_{k} \geqslant s_{k}^{*}>0 .
$$

Докажем, что тогда полюсы $t_{i}$ и $t_{i}^{*}(1 \leqslant i \leqslant k)$ аппроксимаций $p(\mathscr{S}, s)$ и $p\left(\mathscr{S}^{*}, s\right)$ тоже чередуются, причем неравенства строгие (если только $\mathscr{S}$ и $\mathscr{S}^{*}$ не совпадают):

$$
t_{1}>t_{1}^{*}>\cdots>t_{k}>t_{k}^{*}>x>0 .
$$

По построению $p(\mathscr{S}, 0)=p\left(\mathscr{S}^{*}, 0\right)$ и, кроме того,

$$
p\left(\mathscr{S}, s_{j}\right)=I\left(s_{j}\right) \leqslant p\left(\mathscr{S}^{*}, s_{j}\right), \quad p\left(\mathscr{S}^{*}, s_{j}^{*}\right)=I\left(s_{j}^{*}\right) \leqslant p\left(\mathscr{S}, s_{j}^{*}\right), \quad 1 \leqslant j \leqslant k .
$$

Поэтому для функции

$$
\rho(s)=p(\mathscr{S}, s)-p\left(\mathscr{S}^{*}, s\right)=p_{0}-p_{0}^{*}+\sum_{i=1}^{k}\left(\frac{p_{i}}{s+t_{i}}-\frac{p_{i}^{*}}{s+t_{i}^{*}}\right)
$$

сумма $K$ кратностей корней на полуоси $s \geqslant 0$ и даже сумма $K^{\prime}$ кратностей корней на отрезке $\left[0, s_{1}\right]$ не меньше $2 k: K \geqslant K^{\prime} \geqslant 2 k$. Приведем в $(2.21)$ подобные члены, упорядочим полюсы по убыванию и запишем (2.21) в виде

$$
\rho(s)=c_{0}+\sum_{i=1}^{q} \frac{c_{i}}{s+x_{i}}, \quad x_{1}>\cdots>x_{q}>x>0, \quad q \leqslant 2 k .
$$

По теореме Гантмахера-Крейна число П перемен знака в последовательности коэффициентов $c_{0}, \ldots, c_{q}$ не меньше $K$. Так как $\Pi \leqslant q$, отсюда следует, что $q=\Pi=K=K^{\prime}=2 k$, и $(2.20)$ доказано: если полюсы $t_{i}$ и $t_{i}^{*}$ располагаются в любом другом порядке, то П $<2 k$.

В. Взяв малое $\varepsilon>0$, рассмотрим последовательность наборов

$$
\mathscr{S}_{n}=\left\{s_{n, 1}, \ldots, s_{n, k}\right\}, \quad s_{n, 1}>\cdots>s_{n, k}>0, \quad n=1, \ldots, N,
$$

обладаюшую тремя свойствами: 1) $\left.\left.\mathscr{S}_{1}=\mathscr{S}, 2\right) s_{N, 1}<\varepsilon, 3\right)$ точки наборов $\mathscr{S}_{n}$ и $\mathscr{S}_{n+1}(1 \leqslant n<N)$ чередуются: $s_{n, 1}>s_{n+1,1}>\cdots>s_{n, k}>s_{n+1, k}>0$.

Согласно $\mathbf{A}$ для аппроксимаций $p\left(\mathscr{S}_{n}, s\right)$ и $p\left(\mathscr{S}_{n+1}, s\right)$

$$
t_{n, 1}>t_{n+1,1}>\cdots>t_{n, k}>t_{n+1, k}>x>0 .
$$

Отсюда

$$
t_{1}=t_{1,1}>t_{N, 1} .
$$

С. При $\varepsilon \rightarrow 0$ аппроксимация $p\left(\mathscr{S}_{N}, s\right)$, очевидно, стремится к аппроксимации $r_{0}(s)$ (см. (2.9)). Отсюда ввиду $(2.22)$ следует неравенство $t_{1}>t_{01}$. 


\section{§3. Принцип двойственности}

В этом параграфе будет доказана теорема 3 (сформулированная в п. 1.2).

\section{1. Начнем с проверки двух лемм.}

ЛЕмма 2. При любом $x \in[0,1]$ для каждой парьи $\left(b_{0}, d b\right) \in \mathscr{B}=\mathscr{B}(x)$ число $b_{0}$ положительно.

ДокАЗАТЕЛЬСТво. При любом $x \in[0,1]$ функция $I(s)=I(s, x)$ (см. $(1.2)$ ) убьвает при $s \rightarrow \infty$ как $1 / \sqrt{s}$. Согласно (1.6) если $b_{0}=0$, то функция $r\left(b_{0}, d b, s\right)$ убьвает при $s \rightarrow \infty$ как $(b(1)-b(0)) / s$. Таким образом, равенство $b_{0}=0$ противоречит условию $\left(b_{0}, d b\right) \in \mathscr{B}:$ если $b_{0}=0$, то неравенство $r\left(b_{0}, d b, s\right) \geqslant I(s)$ нарушается при больших $s>0$. Значит, $b_{0}>0$ для каждой пары $\left(b_{0}, d b\right) \in \mathscr{B}$.

Лемма 3. В задаче (В) при каждом $x \in(0,1]$ существует такая зависящая от $x$ nара $\left(b_{0}^{\circ}, d b^{\circ}\right) \in \mathscr{B}=\mathscr{B}(x)$, что $\min _{\mathscr{B}} H\left(b_{0}, d b\right)=H\left(b_{0}^{\circ}, d b^{\circ}\right)$.

ДокАЗАТЕЛЬСТво. Будучи положительной и полунепрерывной снизу на $[0,1]$, мажоранта $M(t)$ достигает положительного минимума $M_{0}$ в некоторой точке $t_{0} \in[0,1]$ :

$$
\min _{[0,1]} M(t)=M\left(t_{0}\right)=M_{0}>0 .
$$

Тем самым для каждой пары $\left(b_{0}, d b\right) \in \mathscr{B}$

$$
H\left(b_{0}, d b\right)=b_{0}+\int_{0}^{1} M(t) d b(t) \geqslant b_{0}+\int_{0}^{1} M_{0} d b(t)=b_{0}+M_{0}(b(1)-b(0)) .
$$

Рассмотрим последовательность пар $\left(b_{0, n}, d b_{n}\right) \in \mathscr{B}$, для которой $b_{n}(0)=0$ и

$$
H\left(b_{0, n}, d b_{n}\right) \rightarrow H^{\circ}=\inf _{\mathscr{B}} H\left(b_{0}, d b\right) .
$$

Так как $x>0$, то $H^{\circ}<\infty$ (поскольку для пары $b_{0}=I(0)=2 /(\pi \sqrt{x}), d b=0$ значение $H\left(b_{0}, d b\right)=2 /(\pi \sqrt{x})$ конечно). Поэтому, ввиду $(3.1), b_{n}(1)$ равномерно ограничены, так что (по второй теореме Хелли [10; гл. VI, теорема 3]) из последовательности неубывающих функций $b_{n}(t)$ можно выбрать подпоследовательность, сходящуюся в каждой точке $t \in[0,1]$ к некоторой неубывающей функции $b^{\circ}(t)$. Чтобы не вводить новых обозначений, будем считать, что сама последовательность $b_{n}(t)$ сходится к $b^{\circ}(t)$ и что при этом $b_{0, n} \rightarrow b_{0}^{\circ} \geqslant 0$. Переходя к пределу в неравенствах

$$
r\left(b_{0, n}, d b_{n}, s\right)=b_{0, n}+\int_{0}^{1} \frac{d b_{n}(t)}{s+t} \geqslant I(s), \quad s \geqslant 0,
$$

получаем (по первой теореме Хелли [10; гл. VI, теорема 2]), что

$$
r\left(b_{0}^{\circ}, d b^{\circ}(t), s\right) \geqslant I(s) \text { при всех } s \geqslant 0, \text { т.е. }\left(b_{0}^{\circ}, d b^{\circ}(t)\right) \in \mathscr{B} .
$$

Если бы мажоранта $M(t)$ была непрерывна на $[0,1]$, то (по той же первой теореме Хелли) выполнялось бы равенство

$$
H^{\circ}=H\left(b_{0}^{\circ}, d b^{\circ}\right)
$$


Поскольку $M(t)$ лиш полунепрерывна снизу, то предельный переход

$$
b_{n}(t) \rightarrow b^{\circ}(t), \quad b_{0, n} \rightarrow b_{0}^{\circ}
$$

дает лишш неравенство $H^{\circ} \geqslant H\left(b_{0}^{\circ}, d b^{\circ}\right)$; но случай $H^{\circ}>H\left(b_{0}^{\circ}, d b^{\circ}\right)$ невозможен, так как $H^{\circ}$ - нижняя грань $H\left(b_{0}, d b\right)$ на $\mathscr{B}$; значит, выполняется $(3.2)$ и лемма 3 доказана.

3.2. Первый шаг к доказательству теоремы 3 сделан - по лемме 3 при любом значении параметра $x \in(0,1]$ нижняя грань в задаче $(\mathrm{B})$ достигается: для некоторой зависящей от $x$ парьи $\left(b_{0}^{\circ}, d b^{\circ}\right) \in \mathscr{B}=\mathscr{B}(x)$

$$
H\left(b_{0}^{\circ}, d b^{\circ}\right)=\min _{\mathscr{B}(x)} H\left(b_{0}, d b\right) .
$$

Остается установить, что при любом $x \in(0,1]$ верхняя грань в задаче $(\mathrm{A})$ тоже достигается (на некоторой зависяшей от $x$ мере $d a^{\circ} \in \mathscr{A}$ ) и

$$
h\left(d a^{\circ}, x\right)=\max _{\mathscr{A}} h(d a, x)=\min _{\mathscr{B}(x)} H\left(b_{0}, d b\right)=H\left(b_{0}^{\circ}, d b^{\circ}\right) .
$$

Лемма 4. Множсество $S^{\circ}$ особъх точек пары $\left(b_{0}^{\circ}, d b^{\circ}\right)$ непусто.

ДокАЗАТЕЛЬСтво. При $s \rightarrow \infty$ разность $r\left(b_{0}^{\circ}, d b^{\circ}, s\right)-I(s)$ стремится к $b_{0}^{\circ}$. По лемме 2 число $b_{0}^{\circ}$ положительно. Поэтому если бы при $s \geqslant 0$ выполнялось строгое неравенство $r\left(b_{0}^{\circ}, d b^{\circ}, s\right)>I(s)$, то выполнялось бы и неравенство

$$
r\left(b_{0}^{\circ}, d b^{\circ}, s\right)>I(s)+\varepsilon
$$

(для некоторого $\varepsilon>0$ ) и можно было бы, не выходя из $\mathscr{B}$, уменьшить $b_{0}^{\circ}$ и тем самьм уменњшить $H\left(b_{0}^{\circ}, d b^{\circ}\right)$. Итак, $r\left(b_{0}^{\circ}, d b^{\circ}, s\right)=I(s)$ при некотором $s \geqslant 0$, т.е. множество $S^{\circ}$ особых точек пары $\left(b_{0}^{\circ}, d b^{\circ}\right)$ непусто.

Так как $X_{k}$ из $(1.5)$ стремятся к нулю, то для любого $x \in(0,1]$ найдется такой номер $k$, что $x \geqslant X_{k}$. Тогда согласно $§ 2$ множество $S^{\circ}$ содержит не более $k$ точек.

Тем самьм, $S^{\circ}$ состоит из конечного числа точек для любого $x \in(0,1]$ :

$$
S^{\circ}=\left\{s_{1}^{\circ}, \ldots, s_{m}^{\circ}\right\}
$$

3.3. Сделаем следующий шаг к доказательству теоремы 3 . Напомним определение множества $\mathscr{P}$ (см. п. 1.2):

$$
\mathscr{P}=\left\{\left(b_{0}, d b\right): b_{0} \geqslant 0, d b=d b(t) \geqslant 0, t \in[0,1]\right\}
$$

Введем множество $\widehat{\mathscr{B}}=\widehat{\mathscr{B}}(x) \supset \mathscr{B}(x)$, состоящее из пар $\left(b_{0}, d b\right) \in \mathscr{P}$, для которых $r\left(b_{0}, d b, s\right) \geqslant I(s)$ на $S^{\circ}$ :

$$
\widehat{\mathscr{B}}=\left\{\left(b_{0}, d b\right) \in \mathscr{P}: r\left(b_{0}, d b, s_{j}^{\circ}\right) \geqslant I\left(s_{j}^{\circ}\right), 1 \leqslant j \leqslant m\right\} .
$$

ЛЕмма 5. Справедливо равенство $H\left(b_{0}^{\circ}, d b^{\circ}\right)=\min _{\widehat{\mathscr{B}}} H\left(b_{0}, d b\right)$. 
ДокАЗАТЕЛЬСтво. Множество $\widehat{\mathscr{B}}$ содержит $\mathscr{B}$, поэтому нижняя грань $H\left(b_{0}, d b\right)$ по всем $\left(b_{0}, d b\right) \in \widehat{\mathscr{B}}$ могла бы оказаться меньше числа $H\left(b_{0}^{\circ}, d b^{\circ}\right)$, равного $\min _{\mathscr{B}} H\left(b_{0}, d b\right)$. Допустим, что это действительно так, и приведем это допушение к противоречию.

Согласно принятому допушению найдется такая пара $\left(b_{0}, d b\right) \in \widehat{\mathscr{B}}$, что

$$
H\left(b_{0}, d b\right)<H\left(b_{0}^{\circ}, d b^{\circ}\right) .
$$

Выбирая достаточно малое $\varepsilon>0$ и умножая $\left(b_{0}, d b\right)$ на $1+\varepsilon$, получаем пару $\left(b_{0}^{\star}, d b^{\star}\right)$, для которой, подобно $(3.6), H\left(b_{0}^{\star}, d b^{\star}\right)<H\left(b_{0}^{\circ}, d b^{\circ}\right)$ и, кроме того, во всех точках $s_{j}^{\circ} \in S^{\circ}$, а значит, и в их малых окрестностях $U_{j}^{\circ}, 1 \leqslant j \leqslant m$, выполняется строгое неравенство

$$
r^{\star}(s)=r\left(b_{0}^{\star}, d b^{\star}, s\right)>I(s), \quad s \in U=U_{1}^{\circ} \cup \cdots \cup U_{m}^{\circ} .
$$

Так как $r^{\circ}(s)=r\left(b_{0}^{\circ}, d b^{\circ}, s\right) \geqslant I(s)$ при всех $s \geqslant 0$ и $r^{\circ}(s)-I(s)>$ const $>0$ вне $U$, то при достаточно малом $\delta>0$ для пары

$$
\left(b_{0, \delta}, d b_{\delta}\right)=(1-\delta)\left(b_{0}^{\circ}, d b^{\circ}\right)+\delta\left(b_{0}^{\star}, d b^{\star}\right)
$$

получаем: $r\left(b_{0, \delta}, d b_{\delta}, s\right)-I(s)=(1-\delta)\left[r^{\circ}(s)-I(s)\right]+\delta\left[r^{\star}(s)-I(s)\right]>0$ при всех $s \geqslant 0$, т.е. $\left(b_{0, \delta}, d b_{\delta}\right) \in \mathscr{B}$. Вместе с тем $H\left(b_{0, \delta}, d b_{\delta}\right)$ строго меньше чем $H\left(b_{0}^{\circ}, d b^{\circ}\right)=\min _{\mathscr{B}} H\left(b_{0}, d b\right)$. Полученное противоречие доказывает лемму 5.

СледСтВИЕ. Пусть $\mathscr{B}^{\circ}-$ подмножество $\widehat{\mathscr{B}}$, на котором $r\left(b_{0}, d b, s_{j}^{\circ}\right)=$ $I\left(s_{j}^{\circ}\right)$ nри $s_{j}^{\circ} \in S^{\circ}, 1 \leqslant j \leqslant m$. Тогда $H\left(b_{0}^{\circ}, d b^{\circ}\right)=\min H\left(b_{0}, d b\right)$ по всем $\left(b_{0}, d b\right) \in \mathscr{B}^{\circ}$.

3.4. Теперь займемся геометрией (в соответствии с конструкцией, описанной в конце п. 1.2).

Введем в $\mathbb{R}^{m+1}$ координаты $v_{0}, v_{1}, \ldots, v_{m}$, причем ось $v_{0}$ направим "вверх".

Отнесем к множеству $V$ точки $\left\{v_{0}, v_{1}, \ldots, v_{m}\right\} \in \mathbb{R}^{m+1}$ с координатами

$$
v_{0}=H\left(b_{0}, d b\right), \quad v_{j}=r\left(b_{0}, d b, s_{j}^{\circ}\right), \quad 1 \leqslant j \leqslant m,
$$

где $\left(b_{0}, d b\right)$ - всевозможные пары из $\mathscr{P}$ (см. (3.4)). Так как $\mathscr{P}$ вместе с каждой парой $\left(b_{0}, d b\right)$ содержит все пары $\left(\lambda b_{0}, \lambda d b\right), \lambda>0$, то $V$ - выпуклый конус с вершиной $O$ в начале координат. В частности, он содержит луч $O H$, где $H=\left\{v_{0}^{\circ}, v_{1}^{\circ}, \ldots, v_{m}^{\circ}\right\}$ - точка с координатами

$$
v_{0}^{\circ}=H\left(b_{0}^{\circ}, d b^{\circ}\right), \quad v_{j}^{\circ}=r\left(b_{0}^{\circ}, d b^{\circ}, s_{j}^{\circ}\right)=I\left(s_{j}^{\circ}\right), \quad 1 \leqslant j \leqslant m .
$$

Тем самым любая гиперплоскость, опорная к $V$ в точке $H$, содержит луч $O H$ и, значит, проходит через начало координат.

Через точку $H$ проведем прямую $L$, параллельную оси $v_{0}$, т.е. зададим $L$ уравнениями

$$
v_{j}=v_{j}^{\circ}=I\left(s_{j}^{\circ}\right), \quad 1 \leqslant j \leqslant m .
$$

По следствию из леммы 5 точка $H$ является самой нижней точкой множества $V$ на L. А сама лемма 5 означает (ввиду (3.5)), что $H$ - самая нижняя точка множества $V$ во всем телесном угле

$$
v_{j} \geqslant v_{j}^{\circ}=I\left(s_{j}^{\circ}\right), \quad 1 \leqslant j \leqslant m .
$$

Иными словами, вьпуклый конус $U=\left\{v_{0}<v_{0}^{\circ}, v_{j} \geqslant v_{j}^{\circ}, 1 \leqslant j \leqslant m\right\}$ с вершиной $H$ не пересекается с $V$. 
Лемма 6. Через точку $H$ проходит опорная $\kappa V$ и трансверсальная $\kappa L$ гиперплоскость $\Pi^{\circ}$, которая задается уравнением

$$
v_{0}=\sum_{j=1}^{m} a_{j}^{\circ} v_{j}
$$

с неотрицательными коэффициентами $a_{j}^{\circ}, 1 \leqslant j \leqslant m$.

ДокАЗАТЕЛЬСтво. За $\Pi^{\circ}$ можно принять любую гиперплоскость $\Pi \supset O H$, опорную к $V$ и не пересекаюшуюся с $U$ (а значит, трансверсальную к $L$ и такую, что в $(3.10)$ все $\left.a_{j} \geqslant 0\right)$. Чтобы "увидеть", что такая гиперплоскость сушествует, полезно рассмотреть еще один выпуклый конус $W$ с вершиной $O$, состояший из всех лучей $O Q, Q \in U$. Тогда $\Pi^{\circ}$ - обшая опорная к $V$ и $W$ гиперплоскость, разделяющая их.

Под опорной гиперплоскостью $\Pi^{\circ}$ нет точек из $V$. Ввиду (3.7) и (3.10) это означает, что для любой пары $\left(b_{0}, d b\right)$ из $\mathscr{P}$

$$
H\left(b_{0}, d b\right) \geqslant \sum_{j=1}^{m} a_{j}^{\circ} r\left(b_{0}, d b, s_{j}^{\circ}\right)
$$

а с учетом (1.6) последнее неравенство можно переписать в виде

$$
b_{0}+\int_{0}^{1} M(t) d b(t) \geqslant \sum_{j=1}^{m} a_{j}^{\circ}\left(b_{0}+\int_{0}^{1} \frac{d b(t)}{s_{j}^{\circ}+t}\right)
$$

или

$$
b_{0}\left(1-\sum_{j=1}^{m} a_{j}^{\circ}\right)+\int_{0}^{1}\left(M(t)-\sum_{j=1}^{m} \frac{a_{j}^{\circ}}{s_{j}^{\circ}+t}\right) d b(t) \geqslant 0 .
$$

Лемма 7. Выполняются два неравенства:

$$
\sum_{j=1}^{m} a_{j}^{\circ} \leqslant 1, \quad \sum_{j=1}^{m} \frac{a_{j}^{\circ}}{s_{j}^{\circ}+t} \leqslant M(t), \quad t \in[0,1] .
$$

ДоказАТЕЛЬство. Множество $\mathscr{P}$ (см. (3.4)) включает в себя как пары, содержащие $d b \equiv 0$, так и пары, в которых $b_{0}=0$, a $d b(t)$ сосредоточена в сколь угодно малой окрестности произвольно заданной точки $t_{0} \in[0,1]$. Поэтому: а) в $(3.11)$ оба слагаемых неотрицательны; b) выполняются неравенства (3.12).

ЛЕмма 8. В уравнении (3.10) сумма всех коэффициентов $a_{j}^{\circ}$ равна 1:

$$
\sum_{j=1}^{m} a_{j}^{\circ}=1
$$


ДокАЗАТЕЛЬСТво. Подставляя в (3.10) координаты (3.8) точки $H^{\circ}$, получаем:

$$
H\left(b_{0}^{\circ}, d b^{\circ}\right)=\sum_{j=1}^{m} a_{j}^{\circ} r\left(b_{0}^{\circ}, d b^{\circ}, s_{j}^{\circ}\right)=\sum_{j=1}^{m} a_{j}^{\circ} I\left(s_{j}^{\circ}\right) .
$$

Действуя так же, как при выводе (3.11), из (3.14) получаем

$$
b_{0}^{\circ}\left(1-\sum_{j=1}^{m} a_{j}^{\circ}\right)+\int_{0}^{1}\left(M(t)-\sum_{j=1}^{m} \frac{a_{j}^{\circ}}{s_{j}^{\circ}+t}\right) d b^{\circ}(t)=0 .
$$

Вследствие (3.12) оба слагаемых в (3.15) неотрицательны, поэтому каждое из них равно нулю, а так как (по лемме 2) $b_{0}^{\circ}>0$, то выполняется равенство (3.13).

ЗАмечАниЕ. Обращение в нуль второго слагаемого в (3.15) означает, что мера $d b^{\circ}(t)$ сосредоточена в особых точках меры

$$
d a^{\circ}=d a^{\circ}(s)=\sum_{j=1}^{m} a_{j}^{\circ} \delta\left(s-s_{j}^{\circ}\right) d s ;
$$

сама мера $d a^{\circ}$ сосредоточена (см. конец п. 3.2$)$ в особых точках пары $\left(b_{0}^{\circ}, d b^{\circ}\right)$.

3.5. Теперь легко завершить

ДокАЗАТЕЛЬСтво теоРемы 3 . Докажем, что $\max _{\mathscr{A}} h$ достигается на меpe $d a^{\circ}$. По леммам 6 и 8 мера $d a^{\circ}$ - неотрицательная мера Стилтьеса с интегралом равным 1 ; по лемме 7

$$
g\left(d a^{\circ}, t\right)=\int_{0}^{\infty} \frac{d a^{\circ}(s)}{s+t}=\sum_{j=1}^{m} \frac{a_{j}^{\circ}}{s_{j}^{\circ}+t} \leqslant M(t), \quad t \in[0,1]
$$

т.е. $d a^{\circ} \in \mathscr{A}$. Подставляя (3.16) в формулу $(2.2)$ для $h(d a)$, получаем:

$$
h\left(d a^{\circ}\right)=\int_{0}^{\infty} I(s) d a^{\circ}(s)=\sum_{j=1}^{m} a_{j}^{\circ} I\left(s_{j}^{\circ}\right) .
$$

Сравнивая (3.17) с (3.14), приходим (с учетом (2.3)) к (3.3). Тот же результат следует (см. замечание к лемме 8) и из критерия совпадения $h\left(d a^{\circ}\right)$ и $H\left(b_{0}^{\circ}, d b^{\circ}\right)$ в п. 1.3. Теорема 3 доказана.

\section{§4. Теорема о строгом максимуме}

4.1. Произвольно фиксируем $x \in(0,1]$ и положим $h(d a)=h(d a, x)$. По теоремам 3 и 4 (см. $\S 3$ и п. 1.3) $\sup _{\mathscr{A}} h(d a)$ достигается на некоторой дискретной мере (сосредоточенной максимум в $k$ точках при $x \geqslant X_{k}$ из (1.5)). Докажем, что для всех остальных мер $d a \in \mathscr{A}$ вьполняется строгое неравенство

$$
h(d a)<h^{\circ}=\max _{\mathscr{A}} h(d a) .
$$


Допустим, что вопреки (4.1) $\max _{\mathscr{A}} h(d a)$ достигается на двух различных мерах $d a_{1}, d a_{2} \in \mathscr{A}$. По теореме 4 обе они дискретны:

$$
d a_{1}=d a_{1}(s)=\sum_{j=1}^{P} a_{1, j} \delta\left(s-s_{1, j}\right) d s, \quad d a_{2}=d a_{2}(s)=\sum_{j=1}^{Q} a_{2, j} \delta\left(s-s_{2, j}\right) d s,
$$

так что множество

$$
\left\{t \in[0,1]: \sum_{j=1}^{P} \frac{a_{1, j}}{s_{1, j}+t}=\sum_{j=1}^{Q} \frac{a_{2, j}}{s_{2, j}+t}\right\},
$$

где $g\left(d a_{1}, t\right)=g\left(d a_{2}, t\right)$, содержит лишш конечное число точек.

Воспользуемся следующей очевидной леммой $о$ выпуклости:

Лемма 9. Пусть меры da $a_{1}$ и da $a_{2}$ принадлежат $\mathscr{A}$, и пусть $h\left(d a_{1}\right)=$ $h\left(d a_{2}\right)=h^{\circ}$, тогда при любых $\alpha \geqslant 0, \beta \geqslant 0, \alpha+\beta=1$, мера da $=\alpha d a_{1}+\beta d a_{2}$ тоже принадлежит $\mathscr{A}$ u $h(d a)=h^{\circ}$.

Для двух различных пар $\left(\alpha_{i}, \beta_{i}\right), \alpha_{i}>0, \beta_{i}>0, \alpha_{i}+\beta_{i}=1, i=1,2$, положим $d a_{i}^{\circ}=\alpha_{i} d a_{1}+\beta_{i} d a_{2}$. Каждая из двух функций

$$
g_{i}^{\circ}(t)=g\left(d a_{i}^{\circ}, t\right)=\alpha_{i} \sum_{j=1}^{P} \frac{a_{1, j}}{s_{1, j}+t}+\beta_{i} \sum_{j=1}^{Q} \frac{a_{2, j}}{s_{2, j}+t}, \quad i=1,2,
$$

совпадает с мажорантой $M(t)$ в тех и только тех точках $t$, в которых

$$
g\left(d a_{1}, t\right)=g\left(d a_{2}, t\right)=M(t),
$$

т.е. на подмножестве множества (4.2).

Все точки этого подмножества, т.е. все особые точки меры $d a_{i}^{\circ}, i=1,2$, обозначим через $t_{1}^{\circ}, \ldots, t_{p}^{\circ}$. Тем самьм при $i=1,2$

$g_{i}^{\circ}\left(t_{k}^{\circ}\right)=g\left(d a_{i}^{\circ}, t_{k}^{\circ}\right)=M\left(t_{k}^{\circ}\right), 1 \leqslant k \leqslant p, \quad g_{i}^{\circ}(t)<M(t), t \in[0,1], t \neq t_{1}^{\circ}, \ldots, t_{p}^{\circ}$.

В частности,

$$
g_{1}^{\circ}\left(t_{k}^{\circ}\right)=g_{2}^{\circ}\left(t_{k}^{\circ}\right), \quad 1 \leqslant k \leqslant p .
$$

По принципу двойственности и по критерию из п. 1.3 (см. также п. 2.1) $h^{\circ}=$ $H^{\circ}=H\left(b_{0}^{\circ}, d b^{\circ}\right)$ для некоторой пары $\left(b_{0}^{\circ}, d b^{\circ}\right)$ из $\mathscr{B}$, причем мера $d b^{\circ}$ сосредоточена в точках $t_{1}^{\circ}, \ldots, t_{p}^{\circ}$ :

$$
d b^{\circ}(t)=\sum_{k=1}^{p} b_{k}^{\circ} \delta\left(t-t_{k}^{\circ}\right) d t, \quad b_{k}^{\circ} \geqslant 0, \quad 1 \leqslant k \leqslant p,
$$

а меры $d a_{i}^{\circ}$ - в особых точках $s_{1}^{\circ}, \ldots, s_{n}^{\circ}$ пары $\left(b_{0}^{\circ}, d b^{\circ}\right)$ :

$$
d a_{i}^{\circ}(s)=\sum_{j=1}^{n} a_{i, j}^{\circ} \delta\left(s-s_{j}^{\circ}\right) d s, \quad a_{i, j}^{\circ} \geqslant 0, \quad i=1,2, \quad 1 \leqslant j \leqslant n,
$$

так что

$$
g_{1}^{\circ}(t)-g_{2}^{\circ}(t)=\sum_{j=1}^{n} \frac{a_{1, j}^{\circ}-a_{2, j}^{\circ}}{s_{j}^{\circ}+t}, \quad t \in[0, \infty) .
$$

С учетом (4.3) разность (4.5) имеет не менее $p$ корней. Чтобы привести к противоречию последнее утверждение и тем самым доказать (4.1), остается проверить следуюшие леммы. 
Лемма 10. Разность (4.5) имеет не более $n-2$ корней, так что $p \leqslant n-2$.

ЛЕмма 11. Виполняется неравенство $p \geqslant n-1$.

4.2. Докажем леммы 10 и 11.

ДокАЗАТЕЛЬСтво ЛЕммЫ 10 . Функции $f_{j}(t), 1 \leqslant j \leqslant n$, равные $1 /\left(s_{j}^{\circ}+t\right)$ при $t \in[0, \infty)$ и 1 при $t=\infty$, образуют чебышёвскую систему на $[0, \infty]$. Поэтому функция $F(t)=\sum_{j=1}^{n}\left(a_{1, j}^{\circ}-a_{2, j}^{\circ}\right) f_{j}(t)$ либо тождественно равна нулю (что невозможно, если $\left.d a_{1}^{\circ} \neq d a_{2}^{\circ}\right)$, либо имеет не более $n-1$ корней на $[0, \infty]$. А так как $\sum_{j=1}^{n} a_{i, j}^{\circ}=1, i=1,2$, то $F(\infty)=\sum_{j=1}^{n}\left(a_{1, j}^{\circ}-a_{2, j}^{\circ}\right) f_{j}(\infty)=0$ и, значит, у разности (4.5) не более $n-2$ корней на $[0, \infty)$.

ДокаЗАТЕЛЬСтво ЛЕммы 11. Среди коэффициентов $b_{k}^{\circ}$ в (4.4), возможно, есть нули, пусть для определенности $b_{1}^{\circ}, \ldots, b_{q}^{\circ}>0, b_{q+1}^{\circ}=\cdots=b_{p}^{\circ}=0$ при некотором $q \leqslant p$, так что

$$
d b^{\circ}(t)=\sum_{k=1}^{q} b_{k}^{\circ} \delta\left(t-t_{k}^{\circ}\right) d t
$$

и, следовательно,

$$
r(s)=r\left(b_{0}^{\circ}, d b^{\circ}, s\right)=b_{0}^{\circ}+\sum_{k=1}^{q} \frac{b_{k}^{\circ}}{s+t_{k}^{\circ}} .
$$

Напомним (см. определение $I(s)$ в (1.2)), что

$$
I(s)=I(s, x)=\frac{2}{\pi} \int_{x}^{\infty} \frac{d \sqrt{t}}{s+t}=\int_{x}^{\infty} \frac{d \theta(t)}{s+t} .
$$

Из приводимого доказательства следует, что искомое неравенство труднее всего получить, если $t_{k}^{\circ}$ в $(4.6), 1 \leqslant k \leqslant q$, и $x$ удовлетворяют условию

$$
x<t_{q}^{\circ}<\cdots<t_{1}^{\circ} .
$$

Пусть (4.7) выполняется. Положим $I(s)=I_{0}(s)+I_{1}(s)+\cdots+I_{q}(s)$, где

$$
I_{q}(s)=\int_{x}^{t_{q}^{\circ}} \frac{d \theta(t)}{s+t}, \ldots, \quad I_{1}(s)=\int_{t_{2}^{\circ}}^{t_{1}^{\circ}} \frac{d \theta(t)}{s+t}, \quad I_{0}(s)=\int_{t_{1}^{\circ}}^{\infty} \frac{d \theta(t)}{s+t} .
$$

Тогда с учетом (4.6)

$$
r(s)-I(s)=b_{0}^{\circ}-I_{0}(s)+\frac{b_{1}^{\circ}}{s+t_{1}^{\circ}}-I_{1}(s)+\cdots+\frac{b_{q}^{\circ}}{s+t_{q}^{\circ}}-I_{q}(s) .
$$

По теореме Гантмахера-Крейна сумма $K$ кратностей корней $r(s)-I(s)$ не больше числа П перемен знака в разложении (4.8): $K \leqslant$ П. Так как в (4.8) всего $2 q+2$ слагаемых, то $\Pi \leqslant 2 q+1(\Pi=2 q+1$ при условии $(4.7))$. Так как особые точки $s_{1}^{\circ}>$ $\cdots>s_{n-1}^{\circ}>0$ пары $\left(b_{0}^{\circ}, d b^{\circ}\right)$ являются кратньми корнями $r(s)-I(s)$ (поскольку $r \geqslant I)$ и, кроме того, $r\left(s_{n}^{\circ}\right)=I\left(s_{n}^{\circ}\right)$, то $2 n-1 \leqslant K$. Значит, $2 n-1 \leqslant K \leqslant \Pi \leqslant 2 q+1$ и, следовательно, $n-1 \leqslant q \leqslant p$.

Лемма 11 - а вместе с ней и теорема 5 о строгом максимуме - доказаны. 


\section{§5. Обобщения}

Теоремы, доказанные в $\S \S 1-4$ для меры $d \theta(t)=\frac{2}{\pi} d \sqrt{t}$, допускают обобщение: после модификации определения (1.5) их можно распространить на довольно широкий класс $\mathbb{S}$ неотрицательных мер Стилтьеса $d \theta(t), t \geqslant 0$.

Опишем меры $d \theta(t) \in \mathbb{S}$, а затем обобщим (1.5) и теоремы из $\S \S 1-4$ (опуская некоторые детали, которые нетрудно восстановить, опираясь на $\S \S 1-4)$.

5.1. В пояснении 1 к $\S 2$ был сделан переход от $d \theta(t)$ к $d \rho(t)=\frac{d \theta(t)}{t}, t \geqslant x>0$, а затем к пропорциональной $d \rho(t)$ мере $d \mu(t)$ с интегралом равным 1.

Сделаем теперь “обратный переход”. Пусть $\rho(t)$ - неубьвающая функция на полуоси $t>0$, стремяшаяся к нулю при $t \rightarrow \infty$ (так что интеграл $d \rho(t)$ от $x$ до бесконечности равен $-\rho(x)$ при любом $x>0)$, и пусть интеграл $t d \rho(t)$ от 1 до бесконечности расходится:

$$
\int_{x}^{\infty} d \rho(t)=-\rho(x), \quad \int_{1}^{\infty} t d \rho(t)=\infty
$$

Если $\rho(t)$ удовлетворяет этим условиям, отнесем $d \theta(t)=t d \rho(t)$ к классу $\mathbb{S}$. Всюду далее предполагается, что мера $d \theta(t)=t d \rho(t) \in \mathbb{S}$.

Так как $\rho(t) \rightarrow 0$ при $t \rightarrow \infty$ и не убьвает, то вследствие (5.1) $\rho(t)<0$ при $t>0$. Значит, $\rho(t)$ не является ступенчатой функцией $с$ конечным числом cmynенек и меры $d \rho(t)$ и $d \theta(t)=t d \rho(t)$ обладают следующими свойствами:

(1) они не сосредоточены в конечном числе точек;

(2) $\rho(t) \neq$ const при $t \in(1, \infty)$.

ЗАмечание 1. Разумеется, условие $d \theta(t)=t d \rho(t) \in \mathbb{S}$ накладьвает и другие ограничения на меры $d \theta(t)$, например, “отсекает” быстро растущие $\theta(t)$; свойства (1), (2) выделены особо, чтобы было удобно ссылаться на них.

Положим

$$
I(s, x)=\int_{x}^{\infty} \frac{d \theta(t)}{s+t}=\int_{x}^{\infty} \frac{t d \rho(t)}{s+t}, \quad s \geqslant 0, x \in[0,1], s^{2}+x^{2} \neq 0 .
$$

Для меры $d \theta(t)=t d \rho(t)$ проведем то же построение, что для меры $\frac{2}{\pi} d \sqrt{t}$ в пояснении 1 к $\S 2$. Ввиду свойства (1) верна

Лемма 12. Для любого $x \in(0,1]$, для функиий $\mu(t)$ и $J(s)$,

$\mu(t)=\mu(t, x)=1-\frac{\rho(t)}{\rho(x)}, t \geqslant x, J(s)=J(s, x)=-\frac{I(s, x)}{\rho(x)}=\int_{x}^{\infty} \frac{t d \mu(t)}{s+t}, s \geqslant 0$,

для любого конечного набора точек $\mathscr{S}=\left\{s_{1}, \ldots, s_{k}\right\}, s_{1}>\cdots>s_{k}>0, u$ для дуги $\mathscr{D}$ в $\mathbb{R}^{2 k+1}$, которую задает вектор-функиия

$$
\xi(t)=\left\{\xi_{0}=1, \frac{t}{s_{1}+t}, \frac{-t}{\left(s_{1}+t\right)^{2}}, \ldots, \frac{t}{s_{k}+t}, \frac{-t}{\left(s_{k}+t\right)^{2}}\right\}, \quad t \geqslant x>0,
$$


можсно ( и притом единственным способом) указать $t_{1}>\cdots>t_{k}>x>0$ и поместить в точку $\xi(\infty)$ и в точки $\xi\left(t_{i}\right)$ дуги $\mathscr{D}$ такие положительнье массы $m_{\infty}$ и $m_{i}, 1 \leqslant i \leqslant k, m_{\infty}+m_{1}+\cdots+m_{k}=1$, что иентр этой системь масс совпадет с точкой

$$
C=\left\{J(0), J\left(s_{1}\right), J^{\prime}\left(s_{1}\right), \ldots, J\left(s_{k}\right), J^{\prime}\left(s_{k}\right)\right\}=\int_{x}^{\infty} \xi(t) d \mu(t) .
$$

ЗАмЕчАнИЕ 2. В доказательстве леммы 12 свойство (1) гарантирует несовпадение функций $I(s)=I(s, x)$ из $(5.2)$ с функциями

$$
p(\mathscr{S}, s)=p_{0}+\sum_{i=1}^{k} \frac{p_{i}}{s+t_{i}}, \quad p_{0}=-m_{\infty} \rho(x), \quad p_{i}=-m_{i} t_{i} \rho(x), 1 \leqslant i \leqslant k,
$$

и тем самьм позволяет применить теорему Гантмахера-Крейна при оценке числа корней разности $p(\mathscr{S}, s)-I(s)$.

Для функции (5.3) из леммы 12 следуют равенства, аналогичные (2.7):

$$
p(\mathscr{S}, 0)=I(0), \quad p\left(\mathscr{S}, s_{j}\right)=I\left(s_{j}\right), \quad p^{\prime}\left(\mathscr{S}, s_{j}\right)=I^{\prime}\left(s_{j}\right), \quad 1 \leqslant j \leqslant k .
$$

5.2. Определим множество $\mathscr{B}=\mathscr{B}(x)$ так же, как в п. 1.2 , только вместо $(1.2)$ используем (5.2). Для набора $\mathscr{S}$ повторим рассуждения из пояснения $5 \mathrm{k} \S 2$.

Возьмем малое $\varepsilon>0$ и рассмотрим последовательность наборов

$$
\mathscr{S}_{n}=\left\{s_{n, 1}, \ldots, s_{n, k}\right\}, \quad s_{n, 1}>\cdots>s_{n, k}>0, \quad n=1, \ldots, N,
$$

обладающую тремя свойствами: 1) $\left.\left.\mathscr{S}_{1}=\mathscr{S} ; 2\right) s_{N, 1}<\varepsilon ; 3\right)$ точки наборов $\mathscr{S}_{n}$ и $\mathscr{S}_{n+1}(1 \leqslant n<N)$ чередуются: $s_{n, 1}>s_{n+1,1}>\cdots>s_{n, k}>s_{n+1, k}>0$. Тогда полюсы аппроксимаций $p\left(\mathscr{S}_{n}, s\right)$ и $p\left(\mathscr{S}_{n+1}, s\right)$ вида $(5.3)$ тоже чередуются:

$$
t_{n, 1}>t_{n+1,1}>\cdots>t_{n, k}>t_{n+1, k}>x>0 \text {. }
$$

При $\varepsilon \rightarrow 0$ отсюда следует

ЛЕмма 13. При любом $x \in(0,1]$ существует и единственна пара

$$
\left(b_{k 0}, d b_{k}\right) \in \mathscr{B}(x), \quad d b_{k}=\sum_{i=1}^{k} b_{k i} \delta\left(t-t_{k i}\right) d t, \quad t_{k 1}>\cdots>t_{k k} \geqslant x>0
$$

для которой при $s=0$ функция

$$
r_{k}(s)=r\left(b_{k 0}, d b_{k}, s\right)=b_{k 0}+\sum_{i=1}^{k} \frac{b_{k i}}{s+t_{k i}}
$$

совпадает с $I(s)=I(s, x)$ вместе с $2 k$ производными:

$$
r_{k}^{(j)}(0)=I^{(j)}(0), \quad 0 \leqslant j \leqslant 2 k .
$$

Числа $t_{1}=t_{1}(x, \mathscr{S})$ в (5.3) $и t_{k 1}=t_{k 1}(x)$ в (5.4) связаны неравенством

$$
t_{1}(x, \mathscr{S})>t_{k 1}(x)
$$


С помощью теоремы Гантмахера-Крейна нетрудно доказать, что при любом фиксированном $k$ функция $t_{k 1}(x)$ является неубъвающей функиией $x$ (ср. с $[4 ; \S 12$, комментарий 44]), а при каждом фиксированном $x \in(0,1]$ и любом $k$ выполняется неравенство

$$
t_{k+1,1}(x)>t_{k 1}(x) .
$$

Лемма 14. Для любого $x \in(0,1]$ найдется такое $k$, что $t_{k 1}(x)>1$.

ДокАЗАТЕЛЬСтво. Допустим, что для некоторого $x \in(0,1]$ при любом $k$

$$
t_{k 1}(x) \leqslant 1
$$

Свяжем с каждой парой (5.4) неубьвающую ступенчатую функцию

$$
B_{k}(t)=B_{k}(t, x), \quad t \in[x, \infty], \quad B_{k}(x)=0
$$

со скачком $B_{k 0}=b_{k 0}(x)$ в точке $t=\infty$ и со скачками $B_{k i}=b_{k i}(x) / t_{k i}(x)$ в точках $t_{k i}=t_{k i}(x), 1 \leqslant i \leqslant k, t_{k 1}>\cdots>t_{k k} \geqslant x$. Ввиду (5.5)

$$
\frac{(-1)^{j}}{j !} r_{k}^{(j)}(0)=\int_{x}^{\infty} \frac{d B_{k}(t)}{t^{j}}=\frac{(-1)^{j}}{j !} I^{(j)}(0)=\int_{x}^{\infty} \frac{d \rho(t)}{t^{j}}, 0 \leqslant j \leqslant 2 k .
$$

Ввиду $(5.8)$ и $(5.9)$ функции $B_{k}(t)$ равномерно ограниченъь: так как

$$
r_{k}(0)=\sum_{i=0}^{k} B_{k i}=I(0)=-\rho(x)
$$

то $B_{k}(t) \leqslant-\rho(x)$ при любом $k$. Значит, по второй теореме Хелли из последовательности $B_{k}(t)$ можно выбрать подпоследовательность, сходящуюся в каждой точке $t \in[x, \infty]$ к некоторой неубываюшей функции $B(t)$, причем вследствие (5.8) $B(t)=$ const при $t \in(1, \infty)$.

По первой теореме Хелли с учетом (5.9)

$$
\int_{x}^{\infty} \frac{d B(t)}{t^{j}}=\int_{x}^{\infty} \frac{d \rho(t)}{t^{j}}, \quad j=0,1,2,3, \ldots
$$

Так как система функций $t^{-j}, j \geqslant 0$, полна на $[x, \infty]$, то из (5.10) следует, что $d B(t)$ и $d \rho(t)$ совпадают. Однако это противоречит свойству $(2): B(t)=$ const, a $\rho(t) \neq$ const при $t \in(1, \infty)$. Полученное противоречие доказывает лемму 14 .

5.3. Теперь для любой меры $d \theta(t) \in \mathbb{S}$ можно дать

ОПРЕДЕЛЕНИЕ УНИВЕРСАЛЬНОЙ ПОСЛЕДОВАТЕЛЬНОСТИ $X_{k}$. ПоЛОЖИМ

$$
X_{k}=\inf \left\{x \in(0,1]: t_{k 1}(x) \geqslant 1\right\}, \quad k=1,2,3, \ldots
$$


Из (5.7) нетрудно вывести, что последовательность $X_{k}-$ невозрастающая, а из леммы 14 следует, что $X_{k} \rightarrow 0$ при $k \rightarrow \infty$.

Повторяя рассуждения из п. 2.2 и из пояснения 2 к $\S 2$ и опираясь на неравенство (5.6) и определение (5.11), получаем

ОСНовНОЕ УТВЕРЖДЕНИЕ ОБ ОСОБЫХ ТОЧКАХ. Для $X_{k} u з$ (5.11) npu

$$
x>X_{k}
$$

любая пара $\left(b_{0}, d b\right) \in \mathscr{B}=\mathscr{B}(x)$ имеет максимум $k$ особых точек.

Если функция $\rho(t)$ непрерывна справа, можно заменить inf в (5.11) на min и знак > в (5.12) на $\geqslant$; если функция $\rho(t)$ непрерывна, то $X_{k}$ убъвают, пока $X_{k}>0$ (может случиться, что, начиная с некоторого $k$, все $X_{k}=0$; так будет, например, если $\rho(t)=$ const вблизи нуля).

5.4. Теперь перейдем к обобщению принципа двойственности. В общем случае все построения - те же, что в $\S 3$, нужно лишь внести небольшие изменения в доказательства лемм 2 и 3.

Докажем соотношение, которое используется при проверке леммы 2:

$$
s I(s, 1) \rightarrow \infty \text { при } s \rightarrow \infty .
$$

Пусть $c>0$ - любое число и $C>c$. Ввиду (5.1) найдется столь большое $X$, что $\int_{1}^{X} t d \rho(t)>C$. Отсюда

$$
I(s, 1)=\int_{1}^{\infty} \frac{t d \rho(t)}{s+t}>\int_{1}^{X} \frac{t d \rho(t)}{s+t}>\frac{1}{s+X} \int_{1}^{X} t d \rho(t)>\frac{C}{s+X} .
$$

Поэтому (так как $C>c$ ) найдется такое $S$, что $I(s, 1)>c / s$ при всех $s>S$.

Лемма 2 сразу следует из (5.13), так как $I(s, x) \geqslant I(s, 1)$ при всех $x \in[0,1]$.

В доказательстве леммы 3 по-новому обосновывается неравенство $H^{\circ}<\infty$ : ввиду (5.1) и (5.2) $I(0, x)=-\rho(x)<\infty$ при любом $x \in(0,1]$; поэтому для пары $b_{0}=-\rho(x), d b=0$ значение $H\left(b_{0}, d b\right)$ конечно.

Все остальные построения из $\S 3$ (и из $\S 4$ ) проходят без изменений.

Таким образом, основное утверждение об особых точках, принцип двойственности и теорема о строгом максимуме верны для любой меры $d \theta(t) \in \mathbb{S}$.

В качестве следствия отсюда получаем, что и остальные теоремы из $\S 1-4$ переносятся на меры $d \theta(t) \in \mathbb{S}$, только под $X_{k}$ нужно понимать не убывающую последовательность (1.5), а невозрастающую последовательность (5.11), и в теореме 2 в общем случае в $(1.4)$ отрезок $\left[X_{k}, 1\right]$ нужно заменить полуинтервалом $\left(X_{k}, 1\right]$. 


\section{Список литературы}

1. Гервер М. Л., Кудрявцева Е. А. Новое доказательство теорем об универсальной последовательности и экстремалњных свойствах дискретных мер // УМН. 1997. Т. 52. №6. C. $153-154$

2. Гервер М. Л. Новое в классической задаче обращения годографа // Препринт. МИТП РАН, 1997; // Вопросы геодинамики и сейсмологии. Вычислительная сейсмология. Вып. 30. М: Наука, 1998 (в печати).

3. Гервер M.Л., Кудрявцева $E$. А. Об экстремальных свойствах волноводов с конечным числом слоев в задаче обращения годограффа // Докл. АН. 1997. Т. 356. № 1. С. 25-28.

4. Гервер M.Л., Кудрявиева E. А. Универсальная последовательность в классической задаче обращения годографа // Матем. сб. 1997. Т. 188. №4. С. 3-56.

5. Кронрод A. С. Узлы и веса квадратурных формул. М.: Наука, 1964.

6. Крейн М. Г., Нудельман А. А. Проблема моментов Маркова и экстремальные задачи. М.: Наука, 1973.

7. Карлин C., Стадден В. Чебышёвские системы и их применение в анализе и статистике. М.: Наука, 1976.

8. Гантмахер Ф.Р., Крейн М.Г. Осцилляционные матрицы и ядра и малые колебания механических систем. М.-Л.: ГИТТЛ, 1950.

9. Гервер М. Л., Кудрявцева Е. А. Теорема об отношениях предшествования, генерируемых вполне положительными ядрами // Матем. сб. 1995. Т. 186. № 9. С. 19-44.

10. Колмогоров A.Н., Фомин C. В. Элементы теории функций и функционального анализа. М.: Наука, 1972.

Международный институт теории прогноза землетрясений

Поступила в редакцию и математической геофизики РАН;

16.09.1997

Московский государственнцй университет им. М.В. Ломоносова

E-mail: m.l@gerver.mccme.ru ekudr@nw.math.msu.su 